\title{
Anaesthetists and the reporting of adverse drug reactions
}

CSM Update is a regular monthly column written by members and staff of the Committee on Safety of Medicines to explain and discuss the committee's role in licensing drugs and monitoring adverse effects.

Each year in the UK roughly three million anaesthetics are administered. About $\mathbf{0} \cdot \mathbf{2} \%$ produce untoward reactions, ranging from the trivial to the serious. All anaesthetic procedures carry a risk. In the year that Simpson introduced chloroform, 1847, a death was recorded in a girl called Hannah Green. Thirty years later the British Medical Association set up an investigation to look into the large number of deaths which resulted from the use of chloroform in labour.

SINCE THEN the impressive advance of anaesthetic $\checkmark$ science has yielded many new anaesthetic agents. The skill of anaesthetists has undoubtedly been responsible for the excellent standard of safety of anaesthetics today. Nevertheless, the roughly 2000 yellow card reports on anaesthetics that the CSM has received since 1964 suggest considerable underreporting of adverse reactions.

The Joint Committee of the Association of Anaesthetists and the Faculty of Anaesthetists told the Grahame-Smith working

Adverse reactions to anaesthetics reported on yellow cards 1964-85 (deaths are given in parentheses)



These figures do not take into account the wide variations in use of these agents.

Analysis of 62 reports of jaundice after exposure to halothane reported to the $C S M$ in $1978-85$

\begin{tabular}{ccc}
\hline $\begin{array}{c}\text { No of exposures to } \\
\text { halothane }\end{array}$ & $\begin{array}{c}\text { No of cases of } \\
\text { jaundice }\end{array}$ & $\begin{array}{c}\text { No of deaths from } \\
\text { hepatic necrosis }\end{array}$ \\
\hline 1 & 21 & 6 \\
2 & 32 & 13 \\
3 & 5 & 3 \\
4 & 4 & 2 \\
\hline
\end{tabular}

Numbers are relatively small, but repeated exposure to halothane does seem to increase the likelihood of fatal hepatic necrosis, confirming previous data. party of the CSM on adverse reactions that the yellow card was unsuitable for recording acute reactions in anaesthetic practice, and that view was repeated at the recent Linkman conference in Leicester organised by the Association of Anaesthetists. The reasons given for under-reporting included the fact that many anaesthetists work alone, have no secretary, and travel from hospital to hospital, often to a tight timetable, and the yellow card does not fit easily into their work style. Furthermore, the anaesthetist has learnt to accept as part of normal practice the hypotension, apnoea, bronchospasm, and rashes that may complicate an otherwise smooth anaesthetic. At the Linkman conference Professor John Dundee, professor of anaesthetics at Queen's University, Belfast, and a member of the CSM, put forward several suggestions to improve matters-for example, setting up local arrangements for storing and handling yellow cards, perhaps involving theatre sisters or hospital pharmacists; ensuring that cases of adverse reactions discussed at anaesthetists' meetings were reported to the CSM; teaching juniors about the need to report, and improving record keeping facilities, perhaps using microcomputers.

Etomidate and halothane provide two examples of problems with anaesthetics.

TN 1983 Professor Iain Ledingham recorded an increasing mortality among patients in the intensive therapy unit in the Western Infirmary, Glasgow, and suggested, on the basis of a retrospective review of 428 severely injured patients who had been mechanically ventilated, that there might be a connection between these deaths and the treatment of his patients with long term infusions of etomidate. With the help of the Faculty of Anaesthetists we acquired at very short notice evidence from other centres in the UK. It later became clear that after the administration of etomidate there is a fall in circulating plasma cortisol concentrations, unresponsive to corticotrophin stimulation. This information was rapidly communicated to the profession and followed shortly by an appropriate revision of the data sheet by the manufacturers. This experience underlines the value of studies of trends in morbidity and mortality data and their possible correlation with a change in therapeutic practice. It also provided an example of a rewarding collaboration between the CSM and members of a specialty.

TALOTHANE is probably the most widely used of 1 the volatile general anaesthetics. In 1974, after 130 yellow card reports, the CSM wrote to the profession warning that the administration of halothane is associated with a small risk of jaundice, in some cases fatal, and that the risk is greater if there is repeated exposure to the drug. Fatalities have also occurred in a few children. The message remains true today. The incidence of this complication and its mechanism are still not known, despite many published reviews. The development of unexplained jaundice after a halothane anaesthetic is a contraindication to its later use. There is probably no completely safe interval between administrations, but what constitutes an acceptable period between anaesthetic uses of halothane is now being reviewed. The experience with halothane emphasises the importance of obtaining a drug history, especially when that drug is an anaesthetic. 